\title{
Custom real-time ultrasonic instrumentation for simultaneous mixture and flow analysis of binary gases in the CERN ATLAS experiment
}

\author{
M. Alhroob ${ }^{a}$, M. Battistin ${ }^{\mathrm{b}}$, S. Berry ${ }^{\mathrm{b}}$, A. Bitadze $^{\mathrm{c}}$, P. Bonneau ${ }^{\mathrm{b}}, \mathrm{G}$. Boyd $^{\mathrm{a}}$, O. Crespo-Lopez $^{\mathrm{b}}$, \\ C. Degeorge ${ }^{\mathrm{d}}$, C. Deterre ${ }^{\mathrm{e}}$, B. Di Girolamo ${ }^{\mathrm{b}}$, M. Doubek $^{\mathrm{f}}$, G. Favre $^{\mathrm{b}}$, G. Hallewell $^{\mathrm{g}}$, A. Hasib $^{\mathrm{a}}$, \\ S. Katunin ${ }^{\text {h }}$, D. Lombard ${ }^{\mathrm{b}}$, A. Madsen ${ }^{\mathrm{e}}$, S. McMahon ${ }^{\mathrm{i}}$, K. Nagai ${ }^{\mathrm{j}}$, A. O'Rourke ${ }^{\mathrm{e}, \mathrm{c}, *}$, \\ B. Pearson ${ }^{a}$, D. Robinson ${ }^{k}$, C. Rossi ${ }^{1}$, A. Rozanov ${ }^{g}$, E. Stanecka ${ }^{m}$, M. Strauss ${ }^{a}$, V. Vacek ${ }^{f}$, \\ R. Vaglio ${ }^{\mathrm{b}}$, J. Young ${ }^{\mathrm{a}}$, L. Zwalinski ${ }^{\mathrm{b}}$
}

a Department of Physics and Astronomy, University of Oklahoma, Norman, OK 73019, USA

b CERN, 1211 Geneva 23, Switzerland

' School of Physics and Astronomy, University of Manchester, Oxford Road, Manchester M13 9PL, UK

d Physics Department, Indiana University, Bloomington, IN 47405, USA

e DESY, Notkestraße 85, Hamburg 22607, Germany

${ }^{\mathrm{f}}$ Czech Technical University, Technická 4, 16607 Prague 6, Czech Republic

${ }^{g}$ Centre de Physique des Particules de Marseille, 163 Avenue de Luminy, 13288 Marseille Cedex 09, France

${ }^{\text {h }}$ B.P. Konstantinov Petersburg Nuclear Physics Institute (PNPI), 188300 St. Petersburg, Russia

${ }^{\mathrm{i}}$ Rutherford Appleton Laboratory, Science \& Technology Facilities Council, Chilton, Didcot OX11 OQX, UK

${ }^{\mathrm{j}}$ Department of Physics, Oxford University, Keble Road, Oxford OX1 3RH, UK

${ }^{\mathrm{k}}$ Department of Physics and Astronomy, University of Cambridge, Cambridge, UK

${ }^{1}$ INFN Genova, Via Dodecaneso 33, 16146 Genova, Italy

${ }^{\mathrm{m}}$ Institute of Nuclear Physics PAS, ul. Radzikowskiego 152, 31-342 Kraków, Poland

\section{A R T I C L E I N F O}

\section{Article history:}

Received 21 March 2016

Accepted 29 April 2016

Available online 30 April 2016

\section{Keywords:}

Ultrasonic binary gas analysis

Ultrasonic flowmetry

ATLAS detector control system

Sonar

Evaporative cooling

Thermosiphon operation

\begin{abstract}
A B S T R A C T
Custom ultrasonic instruments have been developed for simultaneous monitoring of binary gas mixture and flow in the ATLAS Inner Detector. Sound transit times are measured in opposite directions in flowing gas. Flow rate and sound velocity are respectively calculated from their difference and average. Gas composition is evaluated in real-time by comparison with a sound velocity/composition database, based on the direct dependence of sound velocity on component concentrations in a mixture at known temperature and pressure. Five devices are integrated into the ATLAS Detector Control System. Three instruments monitor coolant leaks into $\mathrm{N}_{2}$ envelopes of the silicon microstrip and Pixel detectors. Resolutions better than $\pm 2 \times 10^{-5}$ and $\pm 2 \times 10^{-4}$ are seen for $\mathrm{C}_{3} \mathrm{~F}_{8}$ and $\mathrm{CO}_{2}$ leak concentrations in $\mathrm{N}_{2}$ respectively. A fourth instrument detects sub-percent levels of air ingress into the $C_{3} F_{8}$ condenser of the new thermosiphon coolant recirculator. Following extensive studies a fifth instrument was built as an angled sound path flowmeter to measure the high returning $\mathrm{C}_{3} \mathrm{~F}_{8}$ vapour flux $\left(\sim 1.2 \mathrm{kgs}^{-1}\right)$. A precision of $<2.3 \% \mathrm{FS}$ for flows up to $10 \mathrm{~ms}^{-1}$ was demonstrated. These instruments have many potential applications where continuous binary gas composition measurement is required, including hydrocarbon and anaesthetic gas mixtures.
\end{abstract}

(c) 2016 The Authors. Published by Elsevier B.V. This is an open access article under the CC BY license (http://creativecommons.org/licenses/by/4.0/).

\section{Introduction}

Custom ultrasonic ("sonar") instruments have been developed for the measurement of binary gas mixtures. The instruments are used for continuous real-time flowmetry and leak monitoring for the cooling circuits of the Inner Detector of the ATLAS experiment

\footnotetext{
* Corresponding author.

E-mail address: abigail.orourke@desy.de (A. O'Rourke).
}

at the CERN Large Hadron Collider.

ATLAS is a particle physics detector, consisting of a series of sub-detectors arranged around the proton beam collision point. The ATLAS Inner Detector is used for tracking charged particles, and is situated inside a 2 Tesla solenoidal magnetic field. It comprises silicon pixel and microstrip (SCT) sensors, and a Transition Radiation Tracker. The silicon sensors are maintained at a low temperature to mitigate the effects of radiation damage [1-4]. The heat from the silicon substrates and their local electronics is evacuated using an evaporative cooling system based on 


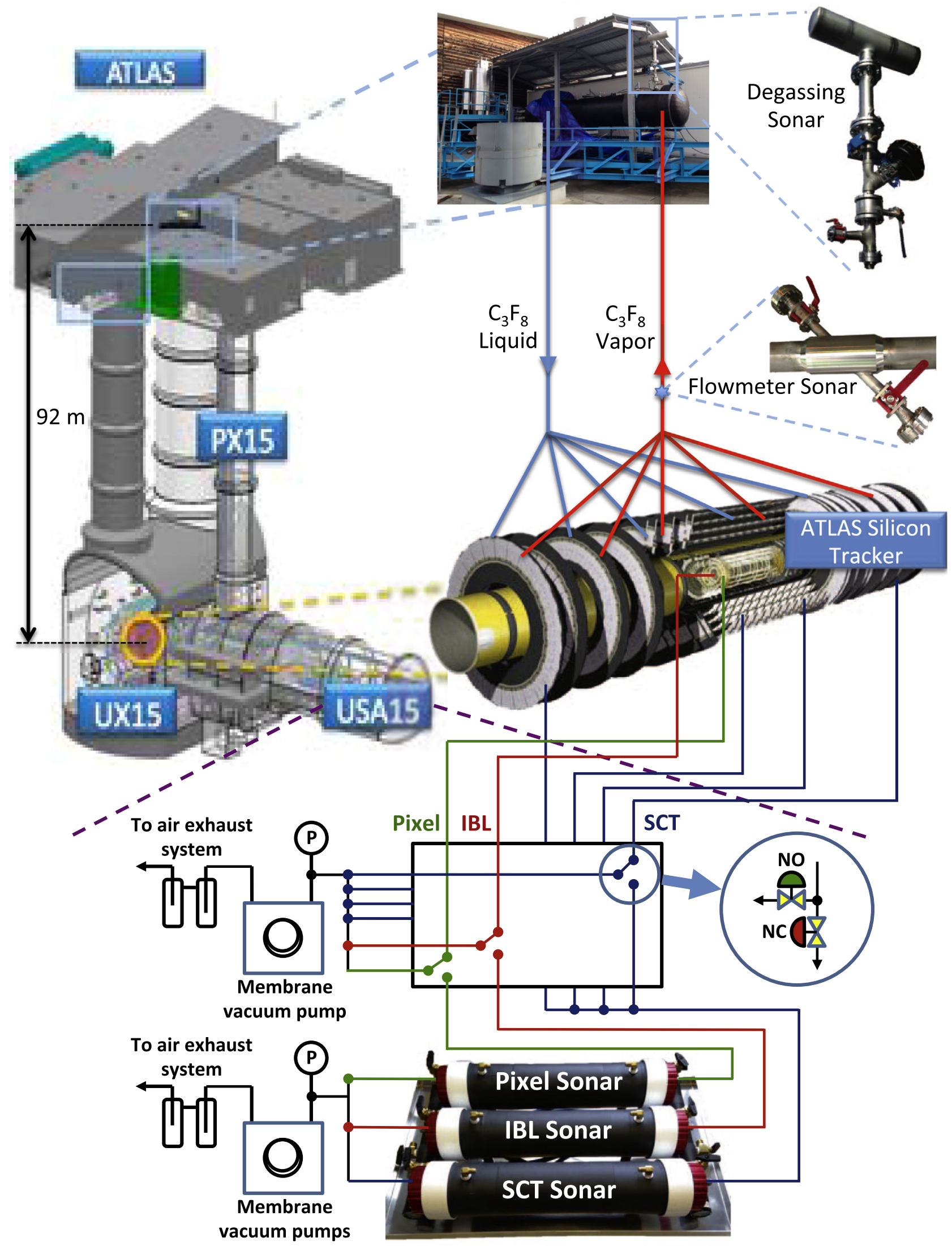

Fig. 1. The locations of the ultrasonic instruments and their connecting structures within the ATLAS inner detector and thermosiphon. 
octafluoropropane $\left(\mathrm{C}_{3} \mathrm{~F}_{8}\right)$ [5]. A new pixel layer, the Insertable B-Layer (IBL), was recently added closest to the beam pipe. The IBL is cooled using a $\mathrm{CO}_{2}$ evaporative cooling system. Three ultrasonic instruments [6-8] monitor the nitrogen-purged anti-humidity envelopes of the SCT, Pixel and IBL subdetectors for leaks from their cooling circuits.

A new $\mathrm{C}_{3} \mathrm{~F}_{8}$ thermosiphon circulation system is being commissioned in $2016[9,10]$ to replace the present compressor-driven system. This utilises the $90 \mathrm{~m}$ depth of the ATLAS experimental cavern to generate the hydrostatic pressure needed to circulate the liquid coolant through the on-detector cooling channels, where it evaporates and returns as vapour to an above-ground condenser. Two further ultrasonic instruments directly monitor the thermosiphon; one measures the flow in the ascendent vapour return tube, while another checks for air ingress into the condenser. The positions of the instruments in the ATLAS installation are shown in Fig. 1.

In this paper, the principle and design of the ultrasonic instruments are described, along with their various applications in the ATLAS cooling system. Their integration into the Detector Control System (DCS) is also discussed.

\section{The instruments}

The sonar instruments use transit time and sound velocity measurements to calculate binary gas compositions and flow rate. Composition measurement in a binary gas mixture exploits the relationship between sound velocity and molar composition at known temperature and pressure. This relationship is illustrated for mixtures of $\mathrm{C}_{3} \mathrm{~F}_{8}$ and $\mathrm{N}_{2}$ at several temperatures in Fig. 2 (see also Section 2.1).

The five installed instruments are based on custom electronics and hardware operating with pairs of $50 \mathrm{kHz}$ capacitive ultrasonic transducers (SensComp model 600) placed within gas enclosures. The transducers operate as transceivers with a DC bias voltage of around $+300 \mathrm{~V}$. In transmit mode, they are excited by down-going $(300 \mathrm{~V}-0 \mathrm{~V})$ square-wave pulses generated from a gated LV pulse output of a DSPIC33F microcontroller. The receiving chain has two amplification stages followed by a comparator, also implemented in the microcontroller. Transmission synchronously starts a $40 \mathrm{MHz}$ transit time clock in the microcontroller, which is stopped by the first pulse crossing the user-definable comparator threshold. A mode of operation based on time-of-flight, rather than resonant frequency, is chosen to allow flowmetry in the same electronics.

Synchronous analogue temperature and pressure measurements use a multiplexed ADC implemented in an Analog Devices

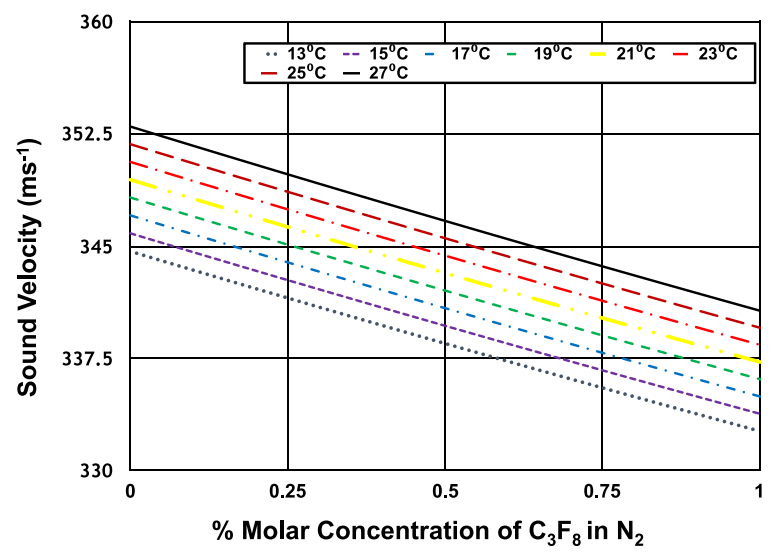

Fig. 2. Examples of the variation of sound velocity with molar concentration of $\mathrm{C}_{3} \mathrm{~F}_{8}$ in $\mathrm{N}_{2}$ (from the online database) at several temperatures, at a pressure of 1000 mbar $_{\text {abs. }}$.

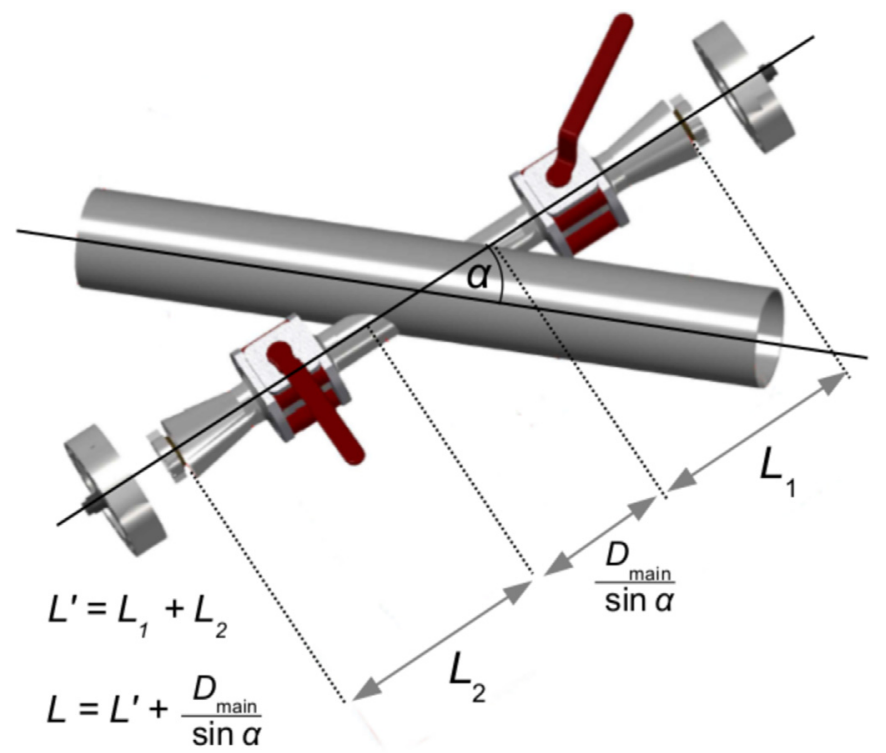

Fig. 3. The ultrasonic flowmeter installed in the $\mathrm{C}_{3} \mathrm{~F}_{8}$ vapour return tube to the thermosiphon condenser. The acoustic path crosses the gas flow at $45^{\circ}$.

$\mathrm{AD} \mu \mathrm{C} 847$ microcontroller. Transit times, temperatures and pressures are averaged, time-stamped and pipelined by a FIFO memory implemented in a dsPIC33F microcontroller for transmission to a SCADA (Supervisory Control and Data Acquisition) computer. Communication uses ModBus TCP/IP over ethernet, implemented using the MBED LPC1768 platform.

Mixture and flow calculations are made on a Dell Poweredge R610 SCADA computer using custom software implemented in SIEMENS SIMATIC WINCC [11] running on LinUX. This software allows control of the instruments and archiving of data.

The installed instruments use two different configurations of acoustic path relative to the gas flow direction: co-axial and angled-crossing. Four instruments in co-axial geometry are optimised for binary concentration measurements in static gas or at low flow. The fifth instrument, shown in Fig. 3, has an acoustic path crossing the gas flow at $45^{\circ}$. It is optimised for flowmetry at high $\mathrm{C}_{3} \mathrm{~F}_{8}$ flow speeds of around $22 \mathrm{~ms}^{-1}$, corresponding to mass flow rates of around $1.2 \mathrm{kgs}^{-1}$.

\subsection{Gas composition analysis}

Binary gas composition is determined by comparison of the measured sound velocity with a stored database of sound velocity as a function of composition for the gas pairs over a range of temperature and pressure covering the process conditions [8].

In an instrument containing either static gas, or where the moving gas fills the entire acoustic path length, $L$, the sound velocity, $v_{s}$, can be determined from the bidirectional transit times $t_{\text {up }}$ and $t_{\text {down }}$ of the sound pulses as

$v_{s}=\frac{2 L\left(t_{\mathrm{up}}+t_{\mathrm{down}}\right)}{4 t_{\mathrm{up}} t_{\text {down }}}$

The three aspirating sonars and the degassing sonar of the thermosiphon condenser (Fig. 1) use a co-axial geometry.

The measured values of temperature, pressure and sound velocity are then compared to a database of measured values created with NIST REFPROP [12] to determine the gas composition. In the case of $\mathrm{C}_{3} \mathrm{~F}_{8}$ in $\mathrm{N}_{2}$, the database is created in temperature and pressure intervals of $0.5^{\circ} \mathrm{C}$ and 20 mbar. An on-line algorithm implemented in WinCC interpolates between the values in the database that are closest to the measured temperature and 
pressure to calculate the molar composition of the binary mixture. This requires an interpolation in two dimensions using four values retrieved from the database

The precision with which the gas composition can be measured depends on the difference between the molecular weights of the two gas components and on the precision of the sound velocity measurement. The latter depends on the precisions of the known transducer spacing $( \pm 0.1 \mathrm{~mm})$, the temperature and pressure in the sonar instrument $\left( \pm 0.1^{\circ} \mathrm{C}\right.$ and $\pm 1 \mathrm{mbar}$, respectively) and the precision of the transit time measurement ( $\pm 25 \mathrm{~ns}$ ). Their combination results in an uncertainty on the measured sound velocity, $\delta v_{s}$, of $\pm 0.025 \mathrm{~ms}^{-1}$. The uncertainty on the gas composition measurement can be defined as $\delta$ (conc) $=\delta v_{s} / m$, where $m$ is the local gradient of the curve of sound velocity against concentration. The average gradient for $\mathrm{C}_{3} \mathrm{~F}_{8}$ in $\mathrm{N}_{2}$ in the interval $0-0.1 \%$ concentration is $-12.55 \mathrm{~ms}^{-1}$ per percent of $\mathrm{C}_{3} \mathrm{~F}_{8}$ (Fig. 2). From this the precision of the concentration measurement is determined to be $\pm 0.002 \%$.

\subsection{Gas flow calculation}

The difference between the transit times in opposite directions is used to calculate the gas flow rate. For the angled ultrasonic flowmeter installed in the return vapour path to the thermosiphon condenser (Fig. 3), the moving gas acoustic path segment is at an angle $\alpha=45^{\circ}$ to the gas flow. The length of this acoustic path is defined as $D_{\text {main }} / \sin \alpha$, where $D_{\text {main }}$ is the diameter of the return gas tube, which is around $135 \mathrm{~mm}$. The remaining length of the sound path, $L^{\prime}$, has components $L_{1}$ and $L_{2}$ on either side of the gas flow tube.

The gas flow velocity, $v_{g}\left(\mathrm{~ms}^{-1}\right)$, can be calculated using the sound velocity measured within the gas, $v_{s}$, as

$v_{\mathrm{g}}=\frac{v_{s}\left(\frac{D_{\text {main }}}{\sin \alpha}+L^{\prime}-v_{s} t_{\text {down }}\right)}{\cos \alpha\left(v_{s} t_{\text {down }}-L^{\prime}\right)}$.

The sound velocity can be calculated as the physical root of

$v_{\mathrm{s}}=\frac{A \pm \sqrt{A^{2}-16 L^{\prime} t_{\mathrm{up}} t_{\mathrm{down}}\left(L^{\prime}+\frac{D_{\text {main }}}{\sin \alpha}\right)}}{4 t_{\mathrm{up}} t_{\text {down }}}$,

where the definition of $A$ is

$A=\left(t_{\text {up }}+t_{\text {down }}\right)\left(2 L^{\prime}+\frac{D_{\text {main }}}{\sin \alpha}\right)$.

An RMS precision of $\pm 2.3 \%$ for full scale (FS) for flows up to $10 \mathrm{~ms}^{-1}$ was demonstrated in pre-installation tests with air [8]. The flowmeter will soon be brought into operation in the closed thermosiphon circuit with $\mathrm{C}_{3} \mathrm{~F}_{8}$.

\section{Application in the ATLAS detector}

\subsection{Implementation}

The configuration of the five sonar instruments deployed in the ATLAS detector is shown in Fig. 1. Three devices aspirate $\mathrm{N}_{2}$ environmental gas from the anti-humidity enclosures of the IBL, Pixel and SCT detectors to monitor the presence of leaks from their respective cooling systems. The SCT environmental volume is monitored at four different points - two in the endcap regions, and two in the barrel region. Gas from each region is monitored for 4 hours at a time in a $16 \mathrm{~h}$ continuous cycle. Two sonar instruments are implemented in the new $\mathrm{C}_{3} \mathrm{~F}_{8}$ thermosiphon coolant recirculation system, which is being commissioned for the Pixel and SCT detectors.
In addition to the ultrasonic flowmeter discussed in Section 2.2, a "degassing" sonar monitors $\mathrm{C}_{3} \mathrm{~F}_{8}$ vapour for possible air contamination in the condenser, reducing thermosiphon performance. Air would be expected to accumulate in the condenser; not only is it the highest part of the system, but also the lowest temperature and pressure point in the cooling circuit. The degassing sonar can trigger the venting of accumulated air when this exceeds safe limits. The air $/ \mathrm{C}_{3} \mathrm{~F}_{8}$ molar composition is measured in the same way as described in Section 2.1.

The precision parameters and the average slope of the sound velocity vs. composition curve in the range $0-30 \%$ air in $\mathrm{C}_{3} \mathrm{~F}_{8}$ over the temperature and pressure range of interest results in a mixture resolution of $\pm 9.4 \times 10^{-4}[6]$.

A user interface and Finite State Machine (FSM) has been created and integrated into the ATLAS DCS. This allows the systems to be monitored and controlled. An alarm system is also in place, which warns of any anomalies, such as a large increase in coolant leak, or hardware errors.

\subsection{Recent measurements}

The aspirating sonar instruments monitored the switch-on of the evaporative cooling systems of the ATLAS Inner Detector silicon tracker during January 2016. The instrument monitoring the $\mathrm{N}_{2}$-purged envelope of the IBL subdetector showed that its ondetector cooling channels were very leak-tight; no increase in $\mathrm{CO}_{2}$ concentration was seen. The instruments monitoring the SCT and Pixel sub-detectors observed changes in the concentration of $\mathrm{C}_{3} \mathrm{~F}_{8}$ coolant in their envelopes.

Fig. 4 shows the concentration of $\mathrm{C}_{3} \mathrm{~F}_{8}$ in the $\mathrm{N}_{2}$ flushing gas around the Pixel detector and its on-detector cooling tubes. A steep rise in $\mathrm{C}_{3} \mathrm{~F}_{8}$ concentration is observed following the simultaneous start of all 88 Pixel detector cooling circuits, followed by a slower increase to a concentration of about $0.14 \%$.

Fig. 5 shows the apparent $\mathrm{C}_{3} \mathrm{~F}_{8}$ concentration in $\mathrm{N}_{2}$ flushing gas in the four enclosures of the SCT subdetector before and after cooling restart. The SCT sonar tube was monitoring gas aspirated from the SCT barrel "level 7" during the cooling restart, revealing a sharp spike in $\mathrm{C}_{3} \mathrm{~F}_{8}$ concentration as the on-detector cooling circuits were initially operated at a higher evaporation pressure before being reduced to the normal operating value of 1.8 bar $_{\mathrm{abs}}$. The apparent $\mathrm{C}_{3} \mathrm{~F}_{8}$ concentration in the SCT barrel increased significantly following cooling restart: from $0.005 \%$ to $0.013 \%$ in gas aspirated from the SCT barrel "level 1", and from $0.006 \%$ to $0.014 \%$ for SCT barrel "level 7". No significant increase was seen in the apparent $\mathrm{C}_{3} \mathrm{~F}_{8}$ concentration in either of the SCT endcaps following

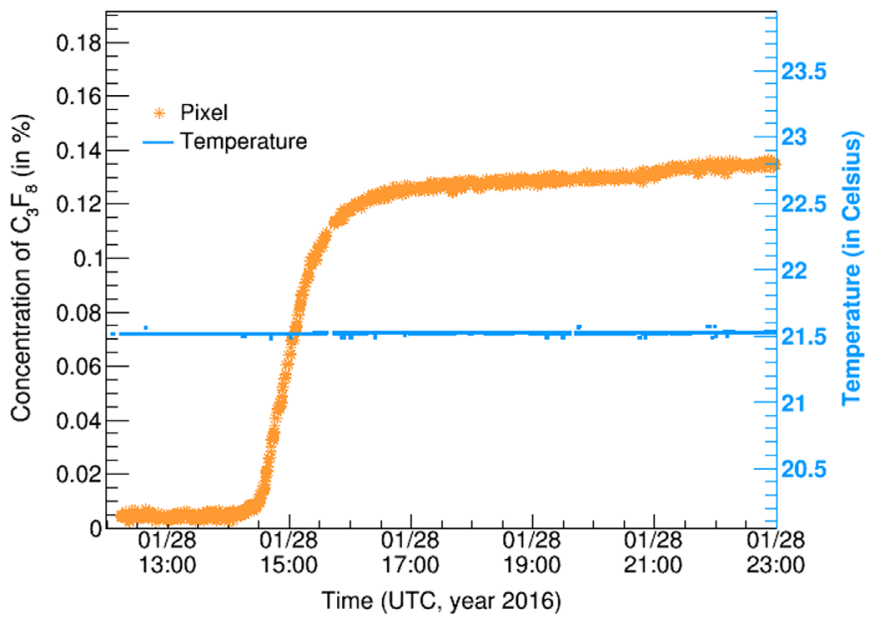

Fig. 4. $\mathrm{C}_{3} \mathrm{~F}_{8}$ concentration in the $\mathrm{N}_{2}$-flushed anti-humidity enclosure of the ATLAS Pixel detector during cooling restart on January 28, 2016. 


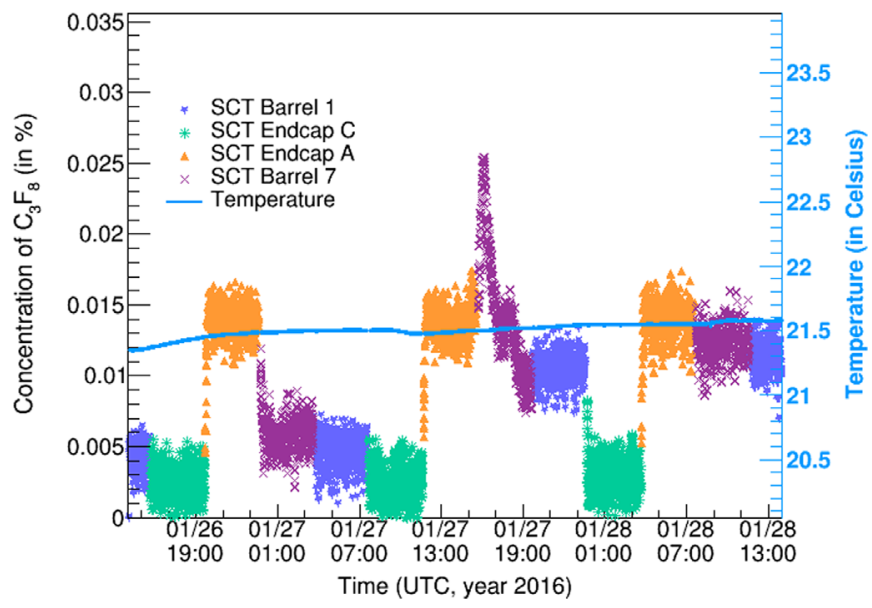

Fig. 5. The concentration of $\mathrm{C}_{3} \mathrm{~F}_{8}$ in $\mathrm{N}_{2}$ for four different regions of the anti-humidity enclosure of the ATLAS SCT detector is observed during the cooling restart around 15:00 UTC on January 27, 2016.

cooling turn on. Endcap " $\mathrm{A}$ " showed a high apparent $\mathrm{C}_{3} \mathrm{~F}_{8}$ concentration before and after the cooling restart, due to a known dry air ingress from the Inner Detector external envelope purge system at the time. Since binary gas analysis is a rapid hypothesisdependent diagnostic, contamination increases in a heavy "search" gas can be mimicked by higher concentrations of a lighter contaminant. Warnings given by the instrument can indicate the need for further investigation with more expensive multi-gas sensitive instrumentation, including gas chromatography.

\section{Summary}

Ultrasonic instrumentation for measurement and real-time monitoring of gas flow and binary gas mixture composition has been developed. Five devices are used in applications related to the evaporative cooling system of the inner detector of the ATLAS experiment, and are integrated into the ATLAS DCS. The monitoring of the cooling of the ATLAS inner detector in January 2016 allowed further validation of the effectiveness of these instruments and the online analysis software. Precisions of $\pm 0.022 \%$ of $\mathrm{CO}_{2}$ in $\mathrm{N}_{2}$ and $\pm 0.002 \%$ of $\mathrm{C}_{3} \mathrm{~F}_{8}$ in $\mathrm{N}_{2}$ have been experimentally verified. An instrument to detect air ingress into the condenser of the new thermosiphon can operate with a molar precision of $\pm 9.4 \times 10^{-4}$. Instruments of this type have other potential applications where continuous real-time flow and binary gas composition measurements are required, for instance hydrocarbon and anaesthetic gas mixture analysis.

\section{Acknowledgements}

A. O'Rourke would like to acknowledge support from the Helmholtz Association.

\section{References}

[1] ATLAS Inner Detector, Technical Design Report, vol. 1, CERN-LHCC-97-16.

[2] ATLAS Inner Detector, Technical Design Report, vol. 2, CERN-LHCC-97-17.

[3] ATLAS Pixel Detector, Technical Design Report, CERN-LHCC-98-13.

[4] G. Beck, G. Viehhauser, Analytic model of thermal runaway in silicon detectors, Nucl. Instrum. Methods A 618 (2010) 131-138, http://dx.doi.org/10.1016/j. nima.2010.02.264.

[5] D. Attree, et al., The evaporative cooling system for the ATLAS inner detector, J. Instrum. 3 (2008) P07003, http://dx doi.org/10.1088/1748-0221/3/07/P07003.

[6] R. Bates, et al., A combined ultrasonic flow meter and binary vapour mixture analyzer for the ATLAS silicon tracker, J. Instrum. 8 (2013) P02006, http://dx. doi.org/10.1088/1748-0221/8/02/P02006 arXiv:1210.4835.

[7] M. Alhroob, et al., Development of a custom on-line ultrasonic vapour analyzer and flow meter for the ATLAS inner detector, with application to Cherenkov and gaseous charged particle detectors, J. Instrum. 10 (03) (2015) C03045 http://dx.doi.org/10.1088/1748-0221/10/03/C03045.

[8] M. Alhroob, et al., Implementations of custom sonar instruments for binary gas mixture and flow analysis in the ATLAS experiment at the CERN LHC, IEEE Trans. Nucl. Sci. (2016) (Submitted for publication).

[9] J. Botehlo-Direito, et al., Full scale thermosiphon technical note, Technical Report, 1083852, CERN, 2010.

[10] M. Battistin, The thermosiphon cooling system of the ATLAS experiment at the CERN Large Hadron Collider, Int. J. Chem. React. Eng. 13 (2015) 511-521, http: //dx.doi.org/10.1515/ijcre-2015-0022.

[11] Siemens, SIMATIC WinCC, 〈http://siemens.com/wincc〉.

[12] E.W. Lemmon, M.L. Huber, M.O. McLinden, NIST Standard Reference Database 23: Reference Fluid Thermodynamic and Transport Properties - REFPROP, Version 9.0, National Institute of Standards and Technology, Standard Reference Data Program, Gaithersburg, 2010. 